\section{Research summarised in this issue}

\section{Relationships between oxidative} stress, haematology and iron profile in anaemic and nonanaemic calves, p 265

Anaemia in calves was associated with more severe oxidative stress than in non-anaemic calves

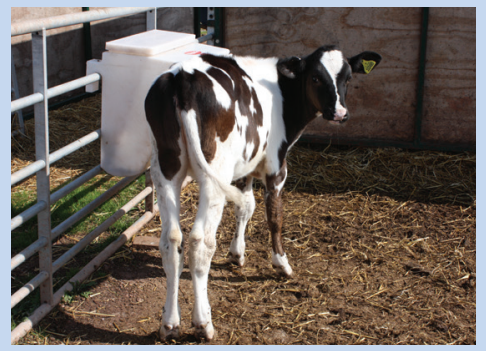

Continuing professional development: researching nontechnical competencies can support cognitive reappraisal and reduced stress in clinicians, p 266 Vets felt more positive about nontechnical competencies after completing a key skills module

\section{Magnitude of reactive}

thrombocytosis and associated clinical conditions in dogs, p 267 Study explores the clinical conditions associated with thrombocytosis in dogs

Full versions of these articles are available at veterinaryrecord. bvapublications

\title{
Anaemia and oxidative stress in calves: an ironclad problem?
}

\section{Fabrizio Ceciliani}

IRON is an essential mineral required for many functions in animal physiology. The iron redox couple reaction mediates the transfer of single electrons through the reversible oxidation/reduction of $\mathrm{Fe}^{2+}$ and $\mathrm{Fe}^{3+}$. This redox cycling is useful for many metabolic pathways essential to life, such as cell cycle progression and division, oxygen transport, mitochondrial activity and immune system function. As a consequence of iron redox reactions, the same metabolic pathways generate reactive oxygen species (ROS) that provide the background for oxidative stress (ie, an imbalance between the production of free radicals and the ability of the body to counteract their harmful effects).

Iron homeostasis is finely tuned: if dysregulated, conditions of iron overload or iron deficiencies can occur. Iron deficiency effects go beyond the well-known importance of iron in the synthesis and function of haemoglobin and myoglobin (and subsequently oxygen transport), by also having further impact on the oxidative status of animals.

Fabrizio Ceciliani, DVM, PhD, Department of Veterinary Medicine, Università degli Studi di Milano Via Celoria 10, 20133 Milano, Italy e-mail address: fabrizio.ceciliani@unimi.it
Iron is a cofactor of enzymes involved in the scavenging of ROS, namely catalase, peroxidase and superoxide dismutase (SOD) ${ }^{1}$. Therefore, iron deficiency reduces organism protection against oxidative stress. On the other hand, excess of iron may have similar effects: iron can bind to the Mn-SODs, with similar affinities and geometries as manganese, ${ }^{2,3}$ resulting in the inactivation of the enzyme. ${ }^{4}$ Moreover, iron has a strong ability to generate highly reactive hydroxyl radicals $(\cdot \mathrm{OH})$ by catalysing hydrogen peroxide, known as Fenton's reaction. These free radicals have the potential to damage cells, organs and tissues.

Iron deficiency occurs frequently during the neonatal period, when iron requirement is greater due to the rapid growth of the organism. ${ }^{5}$ Iron supply for newborns is limited: its concentration in milk is low, and the hepatic reserves come rapidly to an end due to physiological draining. Given that 75 per cent of the body's iron is associated with haemoglobin, iron deficiency limits the synthesis of haemoglobin, resulting in the reduced production of red blood cells (RBC) and, in turn, anaemia. Moreover, the transition from the hypoxic intrauterine life to the extrauterine environment makes newborns very susceptible to oxidative stress. Due

\section{Research in Veterinary Record}

Veterinary Editor in Chief

Professor the Lord Trees

Clinical Research Editor

Tom McNeilly

\section{Editorial Board}

Stephen Baines, Julie Fitzpatrick,

Danièlle Gunn-Moore, John Innes,

Wayne Mcllwraith, Richard Martin,

Chris Oura, Chris Proudman, Stuart Reid,

Susan Rhind, Randolph Richards,

Kenneth Simpson, Polly Taylor, James Wood

\section{Associate Editors}

Alex Corbishley, Richard Drummond,

Felipe Galvao, Darren Green, Tim Greet,

Amy Jennings, Amy Miele, Tim Parkin
Peer-reviewed research papers and short communications in Veterinary Record are published continuously online. The full version of these articles appears on Veterinary Record's website, veterinaryrecord. bvapublications.com, while the print version of the journal contains detailed summaries provided by the author(s).

Once accepted for publication, articles are published in the 'Online First' section of Veterinary Record's website as soon as they have been copyedited, typeset and approved by the author(s). The online articles are available in both PDF and HTML format; each article has a unique doi number and the online version is the definitive publication for citation purposes.

The summaries appearing in the print journal follow a standard format which aims to provide the key findings of the paper in a concise, readable and easily assimilable form. Each gives the title of the paper and author details, and the doi number that links to the full paper online.

This combination of print and online publishing allows Veterinary Record to publish research findings more quickly, while making them more accessible to a wider professional readership. 


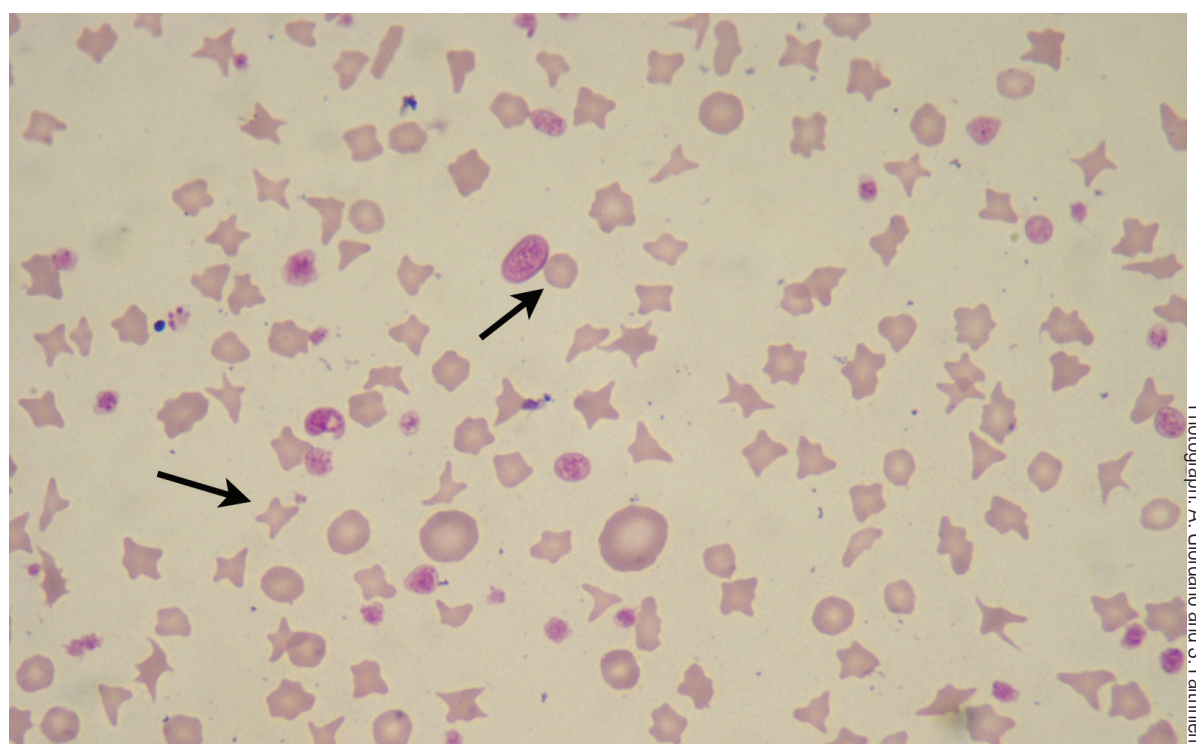

FIG 1: Blood smear from a calf with iron deficiency anaemia. The arrows identify the main features of iron deficiency anaemia, namely: anisocytosis (RBC of unequal size) and microcytosis (RBC with reduced size), due to the iron deficiency and the low capability of synthesis of haemoglobin and poikilocytosis (abnormally shaped RBS), due to the oxidative stress that causes an oxidation of fatty acids within RBC membranes, increasing their deformability and stiffness, is also evident. Diffused hypochromasia (pale RBC) due to a lower percentage of haemoglobin in the RBC is also evident. Wright-Giemsa. x100

to the abundance of polyunsaturated fatty acids within their membranes, erythrocytes are particularly sensitive to oxidative stress, their membranes becoming less deformable and more sensitive to damage, eventually decreasing their life span and worsening the anaemic condition.

Some of the cytopathic effects of iron deficiency on erythrocytes are highlighted in the smears presented in Fig 1, collected from a calf with iron deficiency anaemia. The erythrocytes show the typical features of anaemia, namely, hypochromasia and poikilocytosis. Microcytes and anisocytosis are also present.

The relationship between anaemia and oxidative stress in people has been widely studied, particularly when concerning paediatric diseases. ${ }^{6}$ However, in calves, data on anaemia and oxidative stress are still lacking. One study seeking to address this gap is summarised on p 265 of this week's issue of Veterinary Record. ${ }^{7}$ The study focused on the relationship between iron deficiency and oxidative stress in dairy calves. It was based on previous observations that ROS concentration in the blood of newborn calves was higher than in adult animals, and from this the hypothesis that the susceptibility of calf RBC to oxidative stress was related to an immature defence system against ROS. ${ }^{8}$

The study by Rabjabian and colleagues followed the first month of the calves' lives. Blood samples were collected from 24 to 48 hours after birth and then weekly up to 28 days of age. Animals were divided into two groups (anaemic and non-anaemic) according to haematocrit, haemoglobin content and RBC count. Iron profiles, such as serum iron, total iron binding

\section{What you need to know}

Iron deficiency in calves is related to oxidative stress

Treatment for iron deficiency should consider a supportive therapy with antioxidants

Iron deficiency is related to other apparently distant diseases, such as respiratory distress syndrome in calves and necrotising enterocolitis in piglets, which are part of the same family of diseases defined as 'oxygen radical diseases of neonatology'. Therefore, they should be considered from a therapeutic and prophylactic perspective as a whole

capacity and transferrin, were measured and compared with oxidative stress parameters, such as malondialdehyde (MDA), a lipid peroxidation marker, and ferric-reducing power assay, used to measure the total antioxidant capacity of serum samples.

As expected, iron and transferrin saturation were decreased in anaemic calves, and oxidative stress indicators, in particular haemolysate MDA, were increased. The authors showed that time had a significant effect on both iron and oxidative stress parameters, the difference between the two groups being wider at day 28. Interestingly, the authors also presented evidence that the commercial diets fed to the calves were not adequately meeting the calves' iron needs. The study highlights a potential link between anaemia and oxidative stress, which could potentially be extended to other farm animal species, such as pigs.

Neonatal iron deficiency anaemia is no longer regarded as an individual disease, but is classified under a group of neonatal diseases now collectively defined as 'oxygen radical diseases of neonatology', ${ }^{8}$ which includes respiratory distress syndrome in calves ${ }^{9}$ and necrotising enterocolitis in piglets. ${ }^{10}$

The study pushes the field forward by highlighting the need to investigate the relationship between iron deprivation and the development of oxidative stress in calves, supporting the hypothesis that in cows, as in people, these diseases could be related to the unbalancing of iron homeostasis. The results pave the way to analogous studies in other species, such as pigs, where the selection of genetic lines yielding higher numbers of piglets makes these breeds much more sensitive to iron deficiency, and provide several insights in to the formulation of diets to tackle this issue. Finally, the finding that a relationship does exist between oxidative stress and iron anaemia in calves may mean that calf anaemia could serve as a potential model for studies on oxidative stress in other neonatal diseases in infants.

\section{References}

${ }^{1}$ Matés JM, Pérez-Gómez C \& Núñez De Castro I Antioxidant enzymes and human diseases. Clin Biochem 1999;32:595-603.

Iranzo $\bigcirc$. Manganese complexes displaying superoxide dismutase activity: a balance between different factors. Bioorg Chemistry 2011;39:73-87.

Yamakura F, Kobayashi K, Furukawa S \& Suzuki, Y. In vitro preparation of iron-substituted human manganese superoxide dismutase: possible toxic properties for mitochondria. Free Radic Biol Med 2007;43:423-430.

${ }^{4}$ Jackson TA \& Brunold TC. Combined spectroscopic/ computational studies on Fe- and $\mathrm{Mn}$-dependent superoxide dismutases: insights into second-sphere tuning of active site properties. Acc Chem Res 2004:37:461-470.

Liponski P, Stys A \& Starzynski RR. Molecular insights into the regulation of iron metabolism during the prenatal and early postnatal periods. Cell Mol Life Sci 2013;70:23-38.

Buonocore G, Perrone S. \& Bracci R. Mechanisms involved in the increased hemolysis in the fetus and newborn. Curr Pediatr Rev 2017. doi:10.2174/15733963 13666170718151248

Rajabian F., Mohri M \& Heidarpour M. Relationships between oxidative stress, haematology and iron profile in anaemic and non-anaemic calves. Veterinary Record 2017. doi:10.1136/vr.j104179

Gaál T, Ribiczeyné-Szabó P, Stadler K, Jakus J, Reiczigel J, Kövér et al. Free radicals, lipid peroxidation and the antioxidant system in the blood of cows and newborn calves around calving. Comp Biochem Physiol B Biochem Mol Biol 2006;143: 391-396.

${ }^{9}$ Bleul U. Respiratory distress syndrome in calves. Vet Clin North Am Food Anim Practi 2009;25:179-193.

${ }^{10}$ Jiang P \& Sangild PT. Intestinal proteomics in pig models of necrotising enterocolitis, short bowel syndrome and intrauterine growth restriction. Proteomics Clin Appl 2014;8:700-714

doi: $10.1136 / v r . j 4024$

\section{Submit to Veterinary Record Case Reports for free}

BVA members can now submit cases for free in Veterinary Record Case Reports until the end of 2017. To obtain the discount code please log in to www.bva.co.uk. To submit and view articles visit vetrecordcasereports.bmj.com 


\section{veterinary Anaemia and oxidative stress in calves: an Record ironclad problem?}

Fabrizio Ceciliani

Veterinary Record 2017 181: 263-264

doi: 10.1136/vr.j4024

Updated information and services can be found at:

http://veterinaryrecord.bmj.com/content/181/10/263

\section{These include: \\ References This article cites 8 articles, 0 of which you can access for free at: http://veterinaryrecord.bmj.com/content/181/10/263\#BIBL}

Email alerting Receive free email alerts when new articles cite this article. Sign up in the service box at the top right corner of the online article.

\section{Notes}

To request permissions go to:

http://group.bmj.com/group/rights-licensing/permissions

To order reprints go to:

http://journals.bmj.com/cgi/reprintform

To subscribe to BMJ go to:

http://group.bmj.com/subscribe/ 